\title{
Platelet Oxidative Stress and Antioxidant Nutrients
}

\author{
Bartimoccia $\mathbf{S}^{1}$, Nocella $\mathbf{C}^{1}$, Pastori $\mathbf{D}^{1}$, Pignatelli $\mathbf{P}^{1}$ and Carnevale $\mathbf{R}^{1,2^{*}}$ \\ ${ }^{1}$ Department of Internal Medicine and Medical Specialties, Sapienza University of Rome, Rome, Italy \\ 2 Department of Medico-Surgical Sciences and Biotechnologies, Sapienza University of Rome, Latina, Italy
}

*Corresponding author: Carnevale R, Department of Internal Medicine and Medical Specialties, Sapienza University of Rome, Rome, Italy, Tel: +390649970102; Fax: +390649970102; E-mail: roberto.carnevale@uniroma1.it

Rec date: Oct 21, 2014; Acc date: Nov 24, 2014; Pub date: Nov 26, 2014

Copyright: (c) 2014 Bartimoccia S et al. This is an open-access article distributed under the terms of the Creative Commons Attribution License, which permits unrestricted use, distribution, and reproduction in any medium, provided the original author and source are credited.

\begin{abstract}
Reactive oxidant species (ROS) and intracellular antioxidants are on a sophisticated equilibrium that may lead to cellular damage in case of enhanced production of ROS and/or lowered antioxidant status. Several enzymatic pathways concur to the platelet production of ROS, including NADPH oxidase, myeloperoxidase, xanthine oxidase and uncoupled eNOS. Platelet NADPH oxidase seems to play a crucial role in platelet activation. Pharmacologic approach to reduce platelet activation by the down-regulation of NAPDH oxidase, may represent a future target of antiplatelet drugs. In this context some antioxidant molecules content in food, such as polyphenols, Vitamin E, Vitamin C and PUFAn-3, would be of potential interest.
\end{abstract}

Keywords: Platelet; Oxidative stress; Antioxidant

\section{Abbrevations:}

DNMQ: 2,3-Dimethoxy-1,4-Naphthoquinone; CD40L: CD40 Ligand; Gpiib/Iiia: Glycoprotein Iib/Iiia; Pufan-3: Polyunsaturated Fatty Acid N-3; ROS : Reactive Oxidant Species

\section{Introduction}

Oxidative stress is believed to play a major role in the formation and progression of atherosclerosis, facilitating LDL oxidation within artery wall, which ultimately leads to foam cells formation and atherosclerotic plaque [1]. Thus, growing evidences suggest that oxidative stress is associated with classic atherosclerotic risk factors reflecting a process of systemic inflammation that predisposes to atherosclerosis [2].

In physiological conditions, there is a balance between formation of reactive oxidant species (ROS) and intracellular antioxidant response. The alterations of this equilibrium lead to cellular damage, both in case of enhanced production of ROS, and/or impaired antioxidant status [3]. ROS are physiologic molecules that act as second messengers for cell activation [4]. Several cells are able to produce ROS, such as neutrophil, monocytes, endothelial cells and platelets [5]. Platelets play a key role in the process of athero-thrombosis, via release of inflammatory and pro-thrombotic molecules [6]. The first study demonstrating that platelets were able to generate ROS was published in 1977 by Marcus [7]. Upon activation, platelets produce ROS that are implicated in: 1) propagation of platelet activation by inactivating nitric oxide, 2) releasing of platelet agonists such as ADP, Collagen, Arachidonic Acid giving formation of isoprostanes, Metalloproteinase and ox-LDL and 3) releasing of pro-atherogenic molecules such as CD40L $[8,9]$.

\section{Oxidative Stress and Platelet Activation}

Several enzymatic pathways concur to the platelet production of ROS including NADPH oxidase, myeloperoxidase, xanthine oxidase and uncoupled eNOS [10]. Among them, NADPH oxidase plays a major role, as shown by the almost complete cellular ROS suppression in patients with hereditary deficiency of NOX2, the catalytic sub-unit of the enzyme [11].

There are some evidences suggesting that ROS formation is functionally relevant for platelet activation [12]. It was observed that the reduction of the cytosolic concentration of hydrogen peroxide by catalase inhibited platelet aggregation [13]. More recently, the inhibition of NADPH oxidase by chemical inhibitors, such as diphenyleneiodonium and apocynin, as well as by specific inhibitory peptides, was found to be associated to a significant reduction of calcium mobilization, GPIIb/IIIa opening and, in turn, platelet activation [14]. Moreover, the use of ROS scavengers or inhibitors of $\mathrm{O}^{2-}$ production reduced platelet activation [14]. Consistent with these findings, $\mathrm{O}^{2-}$ scavenger, apocynin and catalase inhibited platelet activation, whereas a ROS donor such as 2,3-dimethoxy-1,4naphthoquinone (DNMQ) enhanced it [14]. Recent studies suggest a more important role of a specific product of arachidonic acid oxidation, namely isoprostanes, in the late phase of platelet activation and thrombus growth [14]. Isoprostanes are produced from nonenzymatic oxidation of arachidonic acid and possess pro-aggregating properties [15]. Isoprostanes are stable compounds and can be measured in urine [16]. Urinary levels of isoprostanes reflect platelet production of isoprostanes [17]. Enhanced levels of isoprostanes have been found in patients at risk for or with cardiovascular diseases [17].

The role of isoprostanes (8-iso-PGF2 $\alpha$ ) has been investigated in the process of platelet recruitment, a model that mimics in vivo the accumulation of platelets at site of vascular injury [18].

NADPH oxidase activation is also implicated in platelet-mediated LDL oxidation [19]; in fact, in presence of native LDL, activated platelets are able to generate ox-LDL, which in turn serve to further propagate platelet activation [1]. 
In addition to pro-oxidant molecules, activated platelets release pro-atherogenic molecules such as CD40 ligand (CD40L) and Glycoprotein IIb/IIIa (GPIIb/IIIa). CD40L is a protein of the tumor necrosis factor family that is implicated in the pathogenesis of atherosclerosis via its inflammatory and prothrombotic properties [20]. Several studies performed in patients with established acute or chronic coronary heart disease documented that plasma levels of sCD40L are predictive of atherosclerotic progression [21-23]. In particular, in atrial fibrillation patients, CD40L levels were inversely associated to adiponectin levels [24] and were associated to cardiovascular events [25] suggesting that lower antioxidant and higher inflammatory conditions are detectable in patients at a higher risk of stroke.

Regarding GPIIb/IIIa, this molecule plays a major role in the regulation of platelet adhesion and aggregation during haemostasis. Several studies demonstrated that there is, in diabetic patients, an increased of platelet surface expression of glycoprotein Ib (GP Ib), which mediates binding to von Willebrand factor, and GP IIb/IIIa, which mediates platelet-fibrin interaction and represents the final common pathway of platelet activation, leading to platelet aggregation $[26,27]$.

\section{Antioxidant Nutrients and Platelet Activation}

Cells have developed a number of defence mechanisms against oxidative damage [28]. Some enzymatic systems, such as superoxide dismutase, catalase, glutathione peroxidase are defined primary antioxidants. In addition, there are non-enzymatic scavenger that can block free radicals such as glutathione, vitamin E, vitamin C, polyphenols defined secondary antioxidants. Foods may represent a natural source of many of these anti-oxidants [8].

\section{Polyphenols}

Polyphenols are a class of molecules [29] present in many components of the Mediterranean diet. The attention paid to polyphenols is related to epidemiological evidence that point out that a diet rich in polyphenols reduces the susceptibility to various diseases such as diabetes, cardiovascular and Alzheiemer diseases [30-32]. The effect of polyphenols is related to their regulatory property on oxidative stress [33], a key element in the development of many diseases.

Polyphenols may also affect platelet function, as shown by data suggesting an inhibitory effect of polyphenol-rich nutrients, i.e, wine, cocoa and extra vergin olive oil [34] on platelet activation [35]. Previous studies have shown that polyphenols such as catechin/ epicatechin in cocoa reduces platelet activity by inhibiting ADP induced expression of the GP IIb-IIIa surface glycoproteins [36]. Also our group has recently shown that acute administration of $40 \mathrm{~g}$ cocoa is associated with inhibition of platelet activation, which was associated with impaired production of platelet isoprostanes via downregulation of platelet NOX2, responsible of ROS production [37]. Moreover in the same paper we demonstrated in vitro that a mix of epicatechin plus catechin is able to decreased of the p47phox translocation that is essential for the activation of NADPH oxidase [37]. Same results were observed with administration of $10 \mathrm{~g}$ of extra virgin olive oil in healthy subjects in addition to a Mediterranean diet meal. In this study we showed that extra virgin olive oil, rich in polyphenols, down-regulated NOX2 activity, pointing to this enzymatic pathway as a mechanism accounting for the antioxidant activity of extra virgin olive oil [1]. Moreover, Ciancarelli et al. showed a decrease in the platelet activation after 4-week supplementation of red wine in 15 healthy male volunteers [38] (Figure 1).

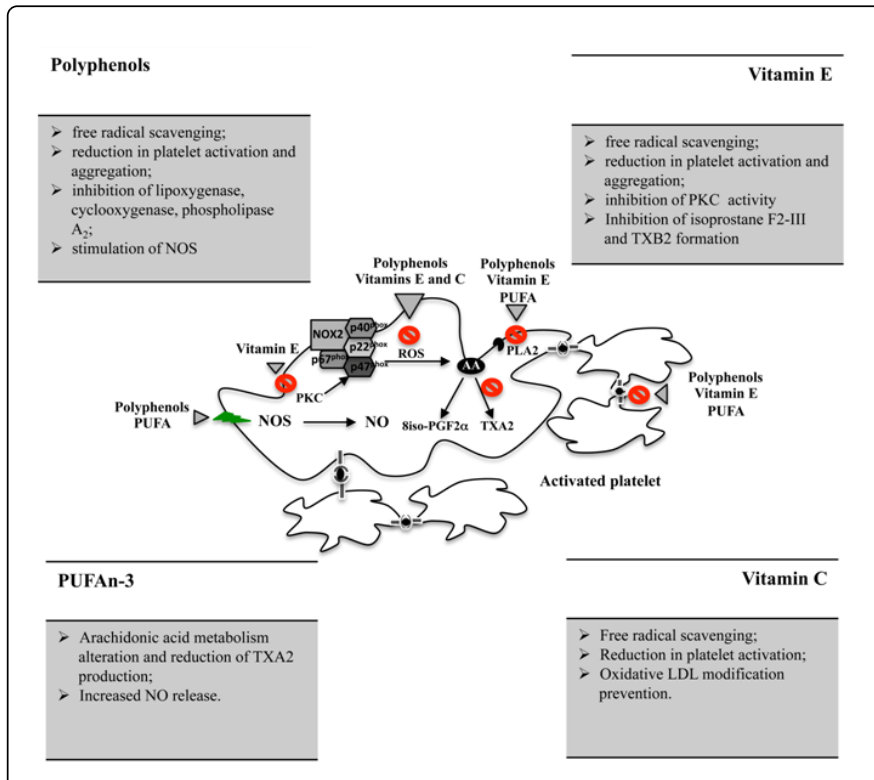

Figure 1: Platelet oxidative stress and antioxidant nutrients; possible mechanism of inhibition

\section{Vitamin E}

Vitamin $\mathrm{E}$ is a chain-breaking antioxidant that prevents the propagation of free radical reaction. As a free radical scavenger, vitamin $\mathrm{E}$ has the role of eliminating lipid soluble free radicals, in conjunction with the network of cellular antioxidant defences, comprising catalases, superoxide dismutases, glutathione-S-transferase and other enzymes, as well as small organic molecules such as Lascorbic acid or b-carotene [39]. Vitamin E has demonstrated to possess anti-platelet and anticoagulant properties [40].

Many studies have demonstrated that vitamin E, in a range of concentration between 50 and $500 \mu \mathrm{M}$, inhibits ex vivo platelet aggregation induced by phorbolemyristate, arachidonic acid and collagen [41]. The mechanism by which vitamin $\mathrm{E}$ inhibits platelet function is controversial, because it is still to be clearly defined if it depends or not on its antioxidant property $[41,42,6]$.

Freedman et al. [41] demonstrated that a daily dosage between 400 and $1200 \mathrm{IU}$ of vitamin $\mathrm{E}$ for 14 days inhibits platelet aggregation elicited by phorbolmyristate and arachidonic acid by interfering with platelet protein kinase C activity. Pignatelli et al. [42] showed that vitamin $\mathrm{E}$ inhibits platelet function through its antioxidant properties, in particular by blocking the capacity of hydrogen peroxide to stimulate platelet arachidonic acid metabolism and the PLC pathway. The interplay between vitamin $\mathrm{E}$ and platelet function has been tested in healthy subjects or in patients at risk of cardiovascular disease such as those with diabetes or dyslipidemia [43] that are associated with enhanced oxidative stress and platelet hyperactivity in vivo [44].

In these studies the authors demonstrated that, after a daily administration of 100 to $600 \mathrm{mg}$ vitamin $\mathrm{E}$ for 8 weeks, there is a significant reduction of both isoprostane F2-III and 11-dehydrothromboxane B2 [41,42], corroborating the hypothesis that oxidative 
Page 3 of 4

stress is implicated in platelet activation and that vitamin $\mathrm{E}$ supplementation may modulate it (Figure 1).

\section{Polyunsaturated fatty acids (PUFAs)}

Polyunsaturated fatty acids (PUFAs) are fatty acids that contain more than one double bond in their backbone [45]. Among the PUFAs, there are two classes, $\omega-3$ and $\omega-6$, in which the first double bond occupies the third or the sixth position from the $\omega$ end, respectively. Linoleic acid ( $\omega-6)$ and alpha-linolenic acid ( $\omega-3)$ are also known as essential fatty acids because they are fundamental for the organism and cannot be synthesized in the human body, so they must be supplied by the diet. PUFAs are present in many species of nuts and vegetable oils [46] and some $\omega-3$ PUFAs are found in fatty fishes.

The antithrombotic properties of fish-derived $\omega$-3 PUFAs have been well-characterized and have been mainly attributed to the incorporation of $\omega-3$ PUFAs into the phospholipid membrane, altering the arachidonic acid metabolism and subsequent reduction in thromboxane (TX) A2 release [47]. As such, $\omega$-3 PUFA compete with arachidonic acid ( $\omega-6$ PUFA) as a substrate for COX enzyme promoting the synthesis of platelet biologically inactive eicosanoids (TXA3) rather than $\omega$-6-derived platelet activator eicosanoids (TXA2) [48]. In addition, $\omega-3$ PUFA have demonstrated to ameliorate vascular function and enhance NO release, thus modulating platelet function [49]. $\alpha$-Linolenic acid (ALA), another $\omega$-3 PUFA found in vegetable oils, has also shown to exert antiplatelet effects. In previously study, it was shown that $\alpha$-linolenic acid incubated with stimulated platelets, reduced platelet $\mathrm{CD} 40 \mathrm{~L}$ expression, $\mathrm{O}^{2-}$ production, $\mathrm{NADPH}$ oxidase activation and p38MAP kinase phosphorylation [50] (Figure 1).

\section{Vitamin C}

Vitamin C (L-ascorbic acid) is an important water soluble vitamin discovered already in the $17^{\text {th }}$ century as the causal factor for scurvy when lacking in the diet, a disease occurring at plasma concentrations below $4 \mu \mathrm{M}$ [51]. Many effects have been attributed to L-ascorbic acid that are the result of its antioxidant, anti-atherogenic, anticarcinogenic and immune-modulatory action [52]. Vitamin C possesses antiplatelet activity and is a direct antioxidant because it quenches superoxide radicals [53]. Based on clinical and epidemiological studies a dietary intake of $100 \mathrm{mg} /$ day of L-ascorbic acid has been suggested to reduce the incidence of mortality from heart disease, stroke and cancer [54] L-Ascorbic acid in vitro prevents the atherogenic, oxidative modification of LDL, shown by a variety of mechanisms. In particular, a previously study has been shown that platelets pre-incubated with vitamin $\mathrm{C}$ inhibit in a dose dependent manner the expression of platelet CD40L via reduction of platelet $\mathrm{O}^{2-}$ [55]. Several studies investigated whether short-term treatment with vitamin $C$, after intravenous $[55,56]$ and oral $[57,58]$ administration affects platelet function. Globally considered, the studies showed inhibition of platelet function after intravenous [51,52] and oral $[57,59]$ administration of vitamin C.

Although the effect achieved after vitamin $\mathrm{C}$ given orally is difficult to explain, the inhibition observed after intravenous vitamin $\mathrm{C}$ administration raises important questions about the role of ROS in platelet activation. Thus, vitamin $\mathrm{C}$ behaves as an antioxidant only if supra-physiological concentration [59]. It is therefore plausible that intravenous administration of vitamin $\mathrm{C}$ actually exerted an antioxidant effect that resulted in platelet ROS inhibition and ultimately reduced platelet activation (Figure 1).

\section{Conclusions}

Several studies support the central role played by ROS in the mechanism of platelet activation. This effect is achieved via multiple pathways, including impaired biosynthesis or inactivation of NO and/or NADPH oxidase activation. Pharmacologic approach that reduces platelet activation may represent a future target of antiplatelet drugs. At this regard some antioxidants contained in foods, such as polyphenols, Vitamin E, Vitamin C or PUFAn-3 would be of potential interest for clinical purposes. To support this theory, randomized interventional trials are necessary to see if they inhibit in vivo platelet aggregation, and if they may eventually be used as part of an antithrombotic treatment in atherosclerotic diseases.

\section{References}

1. Carnevale R, Pignatelli P, Nocella C, Loffredo L, Pastori D, et al. (2014) Extra virgin olive oil blunt post-prandial oxidative stress via NOX2 down-regulation. Atherosclerosis 235: 649-658.

2. Libby P, Ridker PM, Maseri A (2002) Inflammation and atherosclerosis. Circulation 105: 1135-1143.

3. Ray PD, Huang BW, Tsuji Y (2012) Reactive oxygen species (ROS) homeostasis and redox regulation in cellular signaling. Cell Signal 24: 981-990.

4. Violi F, Pignatelli P (2012) Platelet oxidative stress and thrombosis. Thromb Res 129: 378-381.

5. Ide T, Tsutsui H, Kinugawa S, Utsumi H, Kang D, et al. (1999) Mitochondrial electron transport complex $\mathrm{I}$ is a potential source of oxygen free radicals in the failing myocardium. Circ Res 85: 357-363.

6. Pignatelli P, Pulcinelli FM, Lenti L, Gazzaniga PP, Violi F (1998) Hydrogen peroxide is involved in collagen-induced platelet activation. Blood 91: 484-490.

7. Marcus AJ, Silk ST, Safier LB, Ullman HL (1977) Superoxide production and reducing activity in human platelets. J Clin Invest 59: 149-158.

8. Violi F, Pignatelli P, Basili S (2010) Nutrition, supplements, and vitamins in platelet function and bleeding. Circulation 121: 1033-1044.

9. Santos-Martínez MJ, Medina C, Jurasz P, Radomski MW (2008) Role of metalloproteinases in platelet function. Thromb Res 121: 535-542.

10. Krötz F, Sohn HY, Pohl U (2004) Reactive oxygen species: players in the platelet game. Arterioscler Thromb Vasc Biol 24: 1988-1996.

11. Pignatelli P, Sanguigni V, Lenti L, Ferro D, Finocchi A, et al. (2004) gp91phox-dependent expression of platelet CD40 ligand. Circulation 110: 1326-1329.

12. Violi F, Pignatelli P (2014) Platelet NOX, a novel target for antithrombotic treatment. Thromb Haemost 111: 817-823.

13. Del Principe D, Menichelli A, De Matteis W, Di Giulio S, Giordani M, et al. (1991) Hydrogen peroxide is an intermediate in the platelet activation cascade triggered by collagen, but not by thrombin. Thromb Res 62: 365-375.

14. Pignatelli P, Carnevale R, Di Santo S, Bartimoccia S, Sanguigni V, et al. (2011) Inherited human gp91phox deficiency is associated with impaired isoprostane formation and platelet dysfunction. Arterioscle Thromb Vasc Biol 31: 423-434.

15. Ting HJ, Khasawneh FT (2010) Platelet function and Isoprostane biology. Should isoprostanes be the newest member of the orphan-ligand family? J Biomed Sci 17: 24.

16. Tacconelli S, Capone ML, Patrignani P (2010) Measurement of 8-isoprostaglandin F2alpha in biological fluids as a measure of lipid peroxidation. Methods Mol Biol 644: 165-178.

17. Patrignani P, Tacconelli S (2005) Isoprostanes and other markers of peroxidation in atherosclerosis. Biomarkers 10: S24-29.

18. Krötz F, Sohn HY, Gloe T, Zahler S, Riexinger T, et al. (2002) NAD(P)H oxidase-dependent platelet superoxide anion release increases platelet recruitment. Blood 100: 917-924. 
19. Carnevale R, Bartimoccia S, Nocella C, Di Santo S, Loffredo L, et al. (2014) LDL oxidation by platelets propagates platelet activation via an oxidative stress-mediated mechanism. Atherosclerosis 237: 108-116.

20. Schönbeck U, Libby P (2001) CD40 signaling and plaque instability. Circ Res 89: 1092-1103.

21. Heeschen C, Dimmeler S, Hamm CW, van den Brand MJ, Boersma E, et al. (2003) Soluble CD40 ligand in acute coronary syndromes. N Engl J Med 348: 1104-1111.

22. Varo N, de Lemos JA, Libby P, Morrow DA, Murphy SA, et al. (2003) Soluble CD40L: risk prediction after acute coronary syndromes. Circulation 108: 1049-1052.

23. Malarstig A, Lindahl B, Wallentin L, Siegbahn A (2006) Soluble CD40L levels are regulated by the $-3459 \mathrm{~A}>\mathrm{G}$ polymorphism and predict myocardial infarction and the efficacy of antithrombotic treatment in non-ST elevation acute coronary syndrome. Arterioscler Thromb Vasc Biol 26: 1667-1673.

24. Carnevale R, Pastori D, Peruzzi M, De Falco E, Chimenti I, et al. (2014) Total adiponectin is inversely associated with platelet activation and CHA2DS2-VASc score in anticoagulated patients with atrial fibrillation. Mediators Inflamm.

25. Ferro D, Loffredo L, Polimeni L, Fimognari F, Villari P, et al. (2007) Soluble CD40 ligand predicts ischemic stroke and myocardial infarction in patients with nonvalvular atrial fibrillation. Arterioscler Thromb Vasc Biol 27: 2763-2768.

26. Morel O, Kessler L, Ohlmann P, Bareiss P (2010) Diabetes and the platelet: toward new therapeutic paradigms for diabetic atherothrombosis. Atherosclerosis 212: 367-376.

27. Beckman JA, Creager MA, Libby P (2002) Diabetes and atherosclerosis: epidemiology, pathophysiology, and management.JAMA 287: 2570-2581.

28. Kaur R, Kaur J, Mahajan J, Kumar R, Arora S (2014) Oxidative stress-implications, source and its prevention. Environ Sci Pollut Res Int 21: 1599-1613.

29. Tsao R (2010) Chemistry and biochemistry of dietary polyphenols. Nutrients 2: 1231-1246.

30. Dragan S, Andrica F, Serban MC, Timar R (2014) Polyphenols-rich natural products for treatment of diabetes. Curr Med Chem .

31. Khan N, Khymenets O, Urpí-Sardà M, Tulipani S, Garcia-Aloy M, et al. (2014) Cocoa polyphenols and inflammatory markers of cardiovascular disease. Nutrients 6: 844-880.

32. Albarracin SL, Stab B, Casas Z, Sutachan JJ, Samudio I, et al. (2012) Effects of natural antioxidants in neurodegenerative disease. Nutr Neurosci 15: 1-9.

33. Santhakumar AB, Bulmer AC, Singh I (2014) A review of the mechanisms and effectiveness of dietary polyphenols in reducing oxidative stress and thrombotic risk. J Hum Nutr Diet 27: 1-21.

34. Ostertag LM, O'Kennedy N, Kroon PA, Duthie GG, de Roos B (2010) Impact of dietary polyphenols on human platelet function--a critical review of controlled dietary intervention studies. Mol Nutr Food Res 54: 60-81.

35. Pérez-Jiménez J, Neveu V, Vos F, Scalbert A (2010) Identification of the 100 richest dietary sources of polyphenols: an application of the PhenolExplorer database. Eur J Clin Nutr 64 Suppl 3: S112-120.

36. Corti R, Flammer AJ, Hollenberg NK, Lüscher TF (2009) Cocoa and cardiovascular health. Circulation 119: 1433-1441.

37. Loffredo L, Carnevale R, Perri L, Catasca E, Augelletti T, et al. (2011) NOX2-mediated arterial dysfunction in smokers: acute effect of dark chocolate. Heart 97: 1776-1781.

38. TozziCiancarelli MG, Di Massimo C, De Amicis D, Ciancarelli I, Carolei A (2011) Moderate consumption of red wine and human platelet responsiveness. Thromb Res 128: 124-129.

39. Azzi A, Gysin R, Kempná P, Ricciarelli R, Villacorta L, et al. (2003) The role of alpha-tocopherol in preventing disease: from epidemiology to molecular events. Mol Aspects Med 24: 325-336.
40. Pastori D, Carnevale R, Cangemi R, Saliola M, Nocella C, et al. (2013) Vitamin $\mathrm{E}$ serum levels and bleeding risk in patients receiving oral anticoagulant therapy: a retrospective cohort study. J Am Heart Assoc 2: e000364.

41. Freedman JE, Farhat JH, Loscalzo J, Keaney JF Jr (1996) alpha-tocopherol inhibits aggregation of human platelets by a protein kinase C-dependent mechanism. Circulation 94: 2434-2440.

42. Pignatelli P, Pulcinelli FM, Lenti L, Gazzaniga PP, Violi F (1999) Vitamin $\mathrm{E}$ inhibits collagen-induced platelet activation by blunting hydrogen peroxide. Arterioscler Thromb Vasc Biol 19: 2542-2547.

43. Del Ben M, Angelico F, Cangemi R, Loffredo L, Carnevale R, et al. (2012) Moderate weight loss decreases oxidative stress and increases antioxidant status in patients with metabolic syndrome. ISRN Obes 2012: 960427.

44. Davì G, Ciabattoni G, Consoli A, Mezzetti A, Falco A, et al. (1999) In vivo formation of 8-iso-prostaglandin f2alpha and platelet activation in diabetes mellitus: effects of improved metabolic control and vitamin $\mathrm{E}$ supplementation. Circulation 99: 224-229.

45. Jump DB (2002) The biochemistry of n-3 polyunsaturated fatty acids. J Biol Chem 277: 8755-8758.

46. Kris-Etherton PM, Taylor DS, Yu-Poth S, Huth P, Moriarty K, et al. (2000) Polyunsaturated fatty acids in the food chain in the United States. Am J Clin Nutr 71: 179S-88S.

47. Calder PC (2006) n-3 polyunsaturated fatty acids, inflammation, and inflammatory diseases. Am J Clin Nutr 83: 1505S-1519S.

48. von Schacky C, Fischer S, Weber PC (1985) Long-term effects of dietary marine omega-3 fatty acids upon plasma and cellular lipids, platelet function, and eicosanoid formation in humans. J Clin Invest 76 : 1626-1631.

49. Abeywardena MY, Head RJ (2001) Longchain n-3 polyunsaturated fatty acids and blood vessel function. Cardiovasc Res 52: 361-371.

50. Alessandri C, Pignatelli P, Loffredo L, Lenti L, Del Ben M, et al. (2006) Alpha-linolenic acid-rich wheat germ oil decreases oxidative stress and CD40 ligand in patients with mild hypercholesterolemia. Arterioscler Thromb Vasc Biol 26: 2577-2578.

51. Villacorta L, Azzi A, Zingg JM (2007) Regulatory role of vitamins E and $\mathrm{C}$ on extracellular matrix components of the vascular system. Mol Aspects Med 28: 507-537.

52. Grosso G, Bei R, Mistretta A, Marventano S, Calabrese G, et al. (2013) Effects of vitamin $\mathrm{C}$ on health: a review of evidence. Front Biosci (Landmark Ed) 18: 1017-1029.

53. Padayatty SJ, Katz A, Wang Y, Eck P, Kwon O, et al. (2003) Vitamin C as an antioxidant: evaluation of its role in disease prevention. J Am Coll Nutr 22: 18-35.

54. Carr A, Frei B (1999) Does vitamin C act as a pro-oxidant under physiological conditions? FASEB J 13: 1007-1024.

55. Pignatelli P, Sanguigni V, Paola SG, Lo Coco E, Lenti L, et al. (2005) Vitamin C inhibits platelet expression of CD40 ligand. Free Radic Biol Med 38: 1662-1666.

56. Ellis GR, Anderson RA, Chirkov YY, Morris-Thurgood J, Jackson SK, et al. (2001) Acute effects of vitamin C on platelet responsiveness to nitric oxide donors and endothelial function in patients with chronic heart failure. J Cardiovasc Pharmacol 37: 564-570.

57. Morel O, Jesel L, Hugel B, Douchet MP, Zupan M, et al. (2003) Protective effects of vitamin $\mathrm{C}$ on endothelium damage and platelet activation during myocardial infarction in patients with sustained generation of circulating microparticles. J Thromb Haemost 1: 171-177.

58. Padayatty SJ, Sun H, Wang Y, Riordan HD, Hewitt SM, et al. (2004) Vitamin C pharmacokinetics: implications for oral and intravenous use. Ann Intern Med 140: 533-537.

59. Wilkinson IB, Megson IL, MacCallum H, Sogo N, Cockcroft JR, et al. (1999) Oral vitamin C reduces arterial stiffness and platelet aggregation in humans. J Cardiovasc Pharmacol 34: 690-693. 